DOI: https://doi.org/10.46380/rias.v3i2.85

Vol. 3 No. 2, julio-diciembre 2020, pp. 5-17

\title{
El recurso agua en punta Colorada, zona de interés para el sector turístico en Cuba.
}

The water resource in punta Colorada, an area of interest for the tourism sector in Cuba.

O recurso hídrico em punta Colorada, área de interesse para o setor turístico de Cuba.

Carlos Aldana Vilas/Inversiones Gamma S.A., Cuba/carlosaldana64@yahoo.es

Evelio Balado Piedra/Inversiones Gamma S.A., Cuba/eveliobaladopiedra@gmail.com

José Luis Corvea Porras/Inversiones Gamma S.A., Cuba/joselcorvea@gmail.com

Recibido: 6/1/2020 Aceptado: 13/5/2020 Publicado: 30/12/2020

\section{RESUMEN}

La investigación tuvo como objetivo evaluar el recurso agua como elemento clave en una zona de alto potencial turístico, identificada para el desarrollo de un proyecto en la modalidad de golf y marina, el cual implica a punta Colorada en su primera fase. Considerando la distribución, la dinámica y la calidad del agua en el ámbito superficial y subterráneo, se caracterizaron el medio físico del área de estudio y la zona de influencia. Mediante la revisión documental y el uso de las herramientas Flow accumulation de QGIS 2.18.5 y Watershed Maps de Surfer 11, a partir del modelo digital del terreno, se identificaron las direcciones de flujo y acumulación superficial, así como las cuencas locales y su orden de activación. En campaña de campo fueron muestreadas 42 estaciones de aguas terrestres con la medición de todos los parámetros geofísicos in situ y su posterior análisis de laboratorio. Los resultados confirmaron una estrecha relación entre drenaje superficial-humedales; la diferenciación de las lagunas interiores, según su mineralización, y de las aguas subterráneas, según su calidad química. Se concluye que las posibilidades de contaminación, alteración del drenaje y la calidad de las aguas constituyen impactos negativos de dicha inversión en el territorio.

Palabras clave: drenaje superficial, impacto, mineralización

\section{ABSTRACT}

The objective of the research was to evaluate the water resource as a key element in an area with high tourist potential, identified for the development of a project in the golf and marine modality, which involves punta Colorada in its first phase. Considering the distribution, dynamics and quality of the water in the surface and underground environment, the physical 
environment of the study area and the area of influence were characterized. Through the documentary review and the use of the Flow accumulation tools of QGIS 2.18.5 and Watershed Maps of Surfer 11, from the digital terrain model, the directions of flow and surface accumulation were identified, as well as the local basins and their order of activation. In a field campaign, 42 terrestrial water stations were sampled with the measurement of all the geophysical parameters in situ and their subsequent laboratory analysis. The results confirmed a close relationship between surface drainage-wetlands; the differentiation of the interior lagoons, according to their mineralization, and of the groundwater, according to their chemical quality. It is concluded that the possibilities of contamination, alteration of the drainage and the quality of the waters constitute negative impacts of said investment in the territory.

Keywords: impact, mineralization, surface drainage

\section{RESUMO}

O objetivo da pesquisa foi avaliar o recurso hídrico como elemento chave em uma área com alto potencial turístico, identificada para o desenvolvimento de um projeto na modalidade golfe e marinha, que envolve ponta Colorada em sua primeira fase. Considerando a distribuição, dinâmica e qualidade da água nas áreas superficiais e subterrâneas, foram caracterizados o ambiente físico da área de estudo e a área de influência. Por meio da revisão documental e da utilização das ferramentas de Acumulação de Fluxo do QGIS 2.18.5 e Mapas de Bacias Hidrográficas de Surfer 11, a partir do modelo digital de terreno, foram identificados os sentidos de fluxo e acúmulo de superfície, bem como as bacias locais e sua ordem. ativação. Na campanha de campo, 42 estações de água terrestre foram amostradas com a medição de todos os parâmetros geofísicos in situ e sua posterior análise laboratorial. Os resultados confirmaram uma relação estreita entre drenagem superficial-áreas úmidas; a diferenciação das lagoas interiores, segundo a sua mineralização, e das águas subterrâneas, segundo a sua qualidade química. Conclui-se que as possibilidades de contaminação, alteração da drenagem e da qualidade das águas constituem impactos negativos desse investimento no território.

Palavras chave: drenagem superficial, impacto, mineralização

\section{INTRODUCCIÓN}

Desde hace algunos años Cuba se consolida como un destino de alto crecimiento en el área del Caribe y, al mismo tiempo, como un destino turístico singular, especialmente por su contexto geográfico y el carácter insular del territorio. De acuerdo con Salinas et al. (2019), el archipiélago cubano se distingue por la cantidad y variedad de atractivos y recursos de interés para el sector del Turismo, razones que han impulsado un proceso inversionista que se extiende por todo el territorio nacional, con énfasis en las zonas costeras donde se fortalece la modalidad de sol y playa, a la vez que se desarrolla el turismo de golf con un alto potencial y de gran importancia para el país desde el punto de vista económico.

Actualmente, de acuerdo con las indicaciones plasmadas en el Plan de Estado para el Enfrentamiento al Cambio Climático en la República de Cuba, Tarea Vida, las zonas costeras se priorizan por su alta vulnerabilidad, pues el carácter insular 
del territorio determina su interacción permanente con las aguas marinas y costeras, cuyo equilibrio puede afectarse por varias causas de origen humano (Dorticós et al., 2012) y estar condicionado por la creciente actividad turística.

En este escenario, uno de los factores clave es la gestión integrada de los recursos hídricos debido a la alta demanda de agua, su disponibilidad y calidad. Para el caso de las zonas costeras el recurso agua, según Baños et al. (2010), influye en la definición del atractivo o mantenimiento de los destinos turísticos, a la vez que genera gran dependencia tanto por el consumo directo como por su relación con el paisaje y las actividades de ocio, entre las que se incluye el golf (Díaz et al., 2016).

El trabajo que se presenta tuvo como objetivo evaluar el recurso agua como elemento clave en una zona de alto potencial turístico, identificada para el desarrollo de un proyecto en la modalidad de golf y marina, el cual implica a punta Colorada en su primera fase; y cuyo diseño del producto persigue convertirlo en «... un generador económico para el desarrollo de la región, y una vez ejecutado hasta su máximo potencial, demostrará ser una atracción irresistible para turistas de todo el mundo para que descubran Cuba» (Planificación y Arquitectura, 2018).

La evaluación del recurso agua se realiza tanto en el ámbito superficial como subterráneo, teniendo en cuenta las características geográficas del entorno, la distribución y dinámica del recurso, así como los parámetros físicos y químicos que determinan la calidad, a partir de los análisis derivados del muestreo en diversas estaciones identificadas en el inventario y la caracterización de las fuentes de abasto, ubicadas dentro del área de la inversión y la zona de incidencia; lo que permitió identificar los principales impactos en los recursos hídricos del territorio.

\section{MATERIALES Y MÉTODOS}

A partir de la revisión y el análisis de diversas fuentes bibliográficas, informes técnicos y de la cartografía base, se elaboró un esquema de trabajo que incluyó las etapas de gabinete y campo, el que facilitó la caracterización geográfica e hidrológica de la zona de estudio y el diseño de los itinerarios de campo, según las particularidades geográficas y la distribución e identificación de las principales estaciones de muestreo.

Con el uso del Modelo Digital del Terreno (MDT) y la aplicación de las herramientas Flow accumulation de QGIS 2.18.5 y Watershed Maps de Surfer 11, se generó una serie cartográfica con los mapas, lo que permitió la caracterización de la dinámica de las aguas superficiales, su distribución y direcciones de flujo. En campaña de campo se muestrearon en el área de estudio un total de 42 estaciones, de ellas 21 correspondieron directamente a la zona de estudio, siete a una zona propuesta para la construcción de pozos de abasto, enmarcada fuera del perímetro definido para inversión, y 14 vinculadas a la cuenca derivadora del río Salado y el embalse Laguna Grande. Las estaciones incluyeron los tipos de aguas de mar, aguas terrestres superficiales y aguas subterráneas.

En todas ellas fueron precisados sus parámetros geofísicos (in situ), a saber: temperatura, $\mathrm{pH}$, conductividad (SPC), total de sólidos disueltos (TDS) y salinidad. En el caso de los pozos se incluyó, además, el nivel estático (NE). El $43 \%$ (18 estaciones) de las aguas en las 42 estaciones fueron colectadas para el análisis de laboratorio, a las cuales se les realizó la determinación de 
macroconstituyentes, nutrientes nitrogenados, metales y microelementos disueltos.

Los datos obtenidos permitieron determinar el régimen y la calidad de las aguas en la zona de estudio, así como un inventario y caracterización de las fuentes de abasto ubicadas dentro del área, del sistema lacustre de punta Colorada, aguas de mar y aguas subterráneas, además de la relación de los impactos en los recursos hídricos asociados a la intervención prevista en el territorio.

\section{RESULTADOS Y DISCUSIÓN}

\section{Caracterización geográfica e hidrológica}

El área de estudio, localizada en el extremo occidental de Cuba, al noroeste de la provincia Pinar del Río, ocupa un sector del litoral del municipio Sandino (figura 1). Su límite oeste es la bahía de Guadiana y al norte el municipio Mantua.

El sustrato conformado por rocas terrígenas de origen aluvial continental, que afloran hacia el este de dicha región, pertenece a la Formación Guane (Peñalver et al., 2001), representada por arenas y arcillas arenosas. Los sedimentos aluviales asociados al cauce de los ríos están definidos por arenas cuarcíticas y arenas arcillosas, mientras que las arenas de punta Colorada están formadas por granos de cuarzo muy finos subredondeados, transparentes, mezclados con escasos fragmentos de material ferruginoso y partículas de arcillas, que han favorecido su color crema arcilloso, dándole el nombre a la zona (Balado, 2018).

Figura 1. Esquema de localización geográfica del área de estudio.

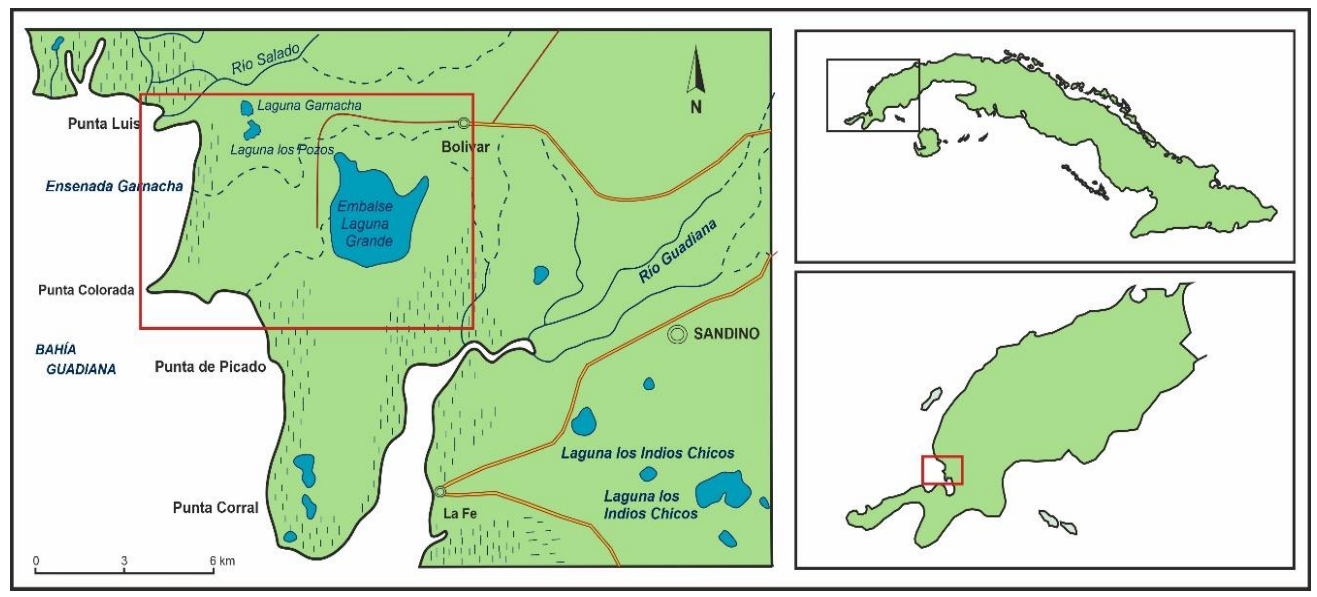

Fuente: Ibarra (1978).

Constituye punta Colorada una pequeña península formada en una llanura de tipo fluvio-marina deltaica, plana y parcialmente cenagosa (Portela et al., 1989). En el extremo oeste sometida a una dinámica litoral estacional que ha provocado cambios en su morfología por la alternancia de períodos acumulativos y erosivos. Hacia el interior, la Ilanura es ligeramente ondulada con mayores alturas. En este entorno se estableció una red de estaciones para el muestreo de las aguas, el que se muestra en la figura 2. 
Figura 2. Esquema de ubicación de estaciones de muestreo.

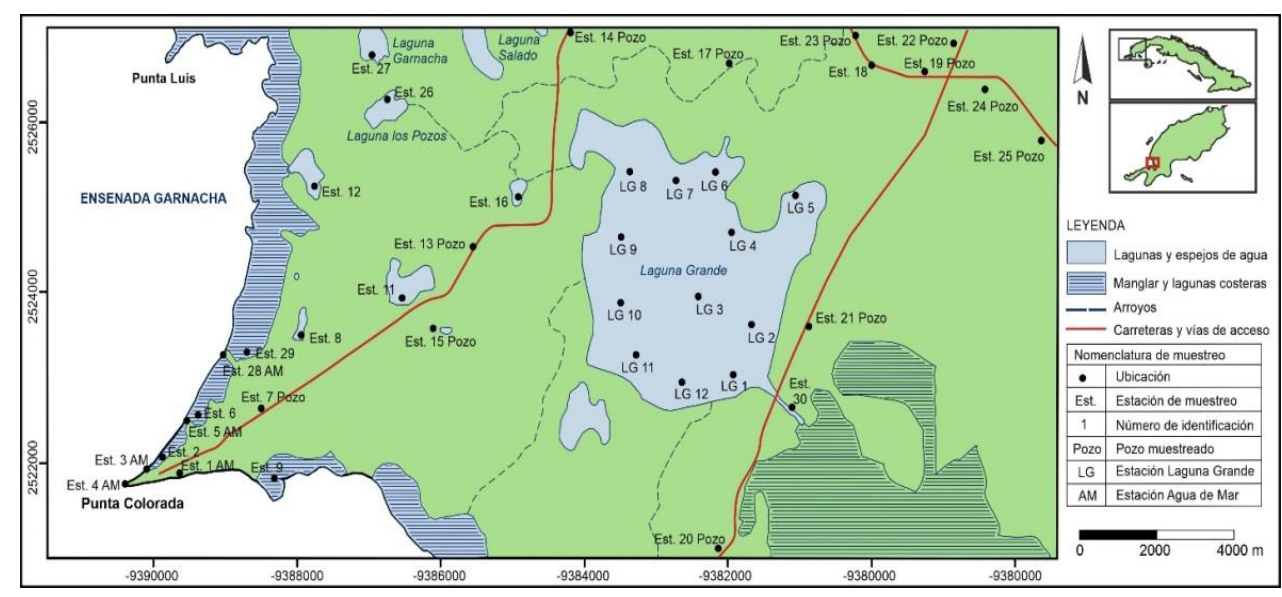

Fuente: Elaboración propia.

\section{Aguas terrestres superficiales}

El mapa altimétrico generado a partir del Modelo Digital del Terreno (MDT) permitió la definición de una red de drenaje de microcuencas con escorrentía eventual y en muy pocos casos, estacional, a pesar de ser una región de paisaje llano muy plano hacia la costa, con pendientes despreciables en la faja litoral (figura 3 ).

Figura 3. Mapa altimétrico con direcciones principales de flujo (izquierda) y mapa de la red de drenaje de aguas superficiales (derecha)
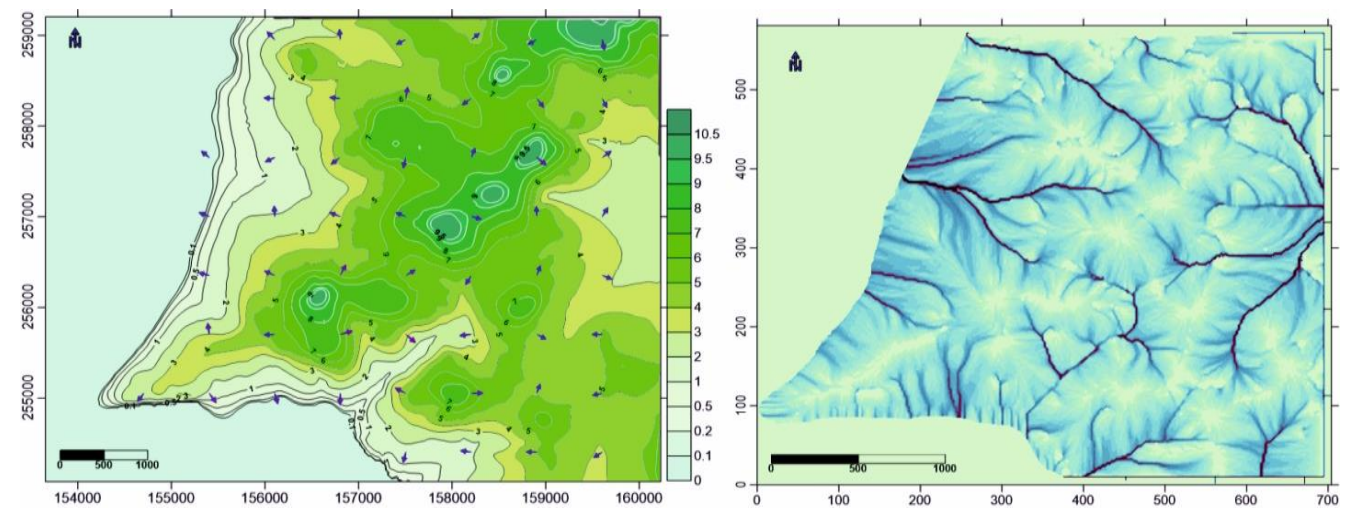

Fuente: Inversiones Gamma (2018).

La herramienta Watershed (Surfer 11), que usa el algoritmo de punto de vertido de ocho direcciones para calcular la dirección del flujo en cada nodo de los archivos Grig, obtenidos del MDT, permitió definir las microcuencas y su orden de activación (figura 4). Lo más significativo de esta dinámica se puede observar en el extremo noroeste del embalse Laguna Grande (microcuencas 3 y 5), el cual capta agua hacia 
la presa en período seco y aporta agua a la vertiente oeste en eventos extremos, donde llega a invertirse el flujo, cuya magnitud de vertido no solo corta la carretera hacia punta Colorada, sino que aporta a los caudales de las lagunas Garnacha y Los Pozos.

Figura 4. Principales microcuencas superficiales del área de estudio en orden de activación hasta 5

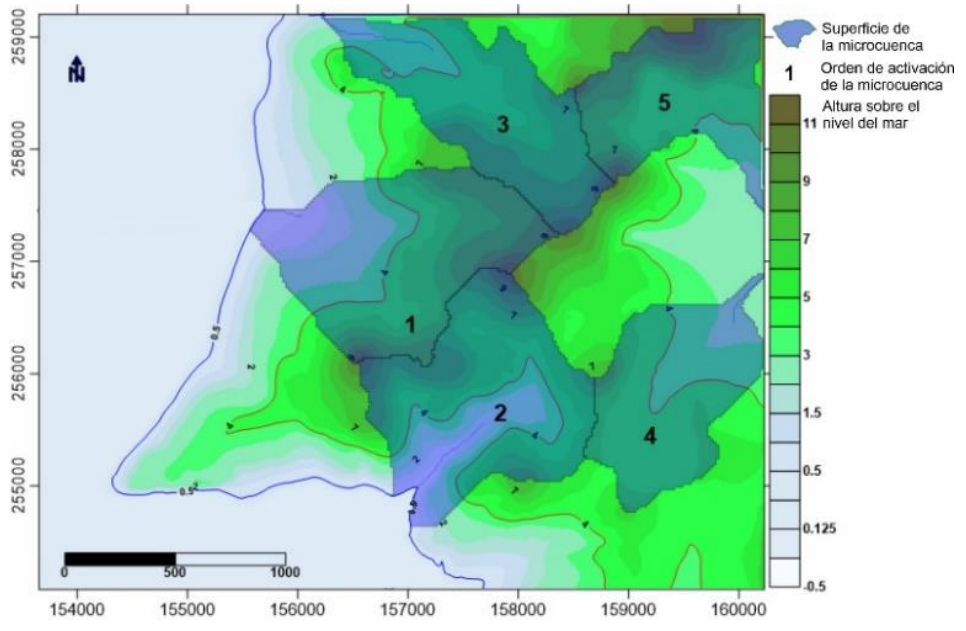

Fuente: Inversiones Gamma (2018).

Inventario y caracterización de las fuentes de abasto ubicadas dentro del área de impacto

En el sector de la cuenca superficial que tributa al embalse Laguna Grande, principal cuerpo de agua de la zona, fueron muestreadas 14 estaciones, de ellas 12 en el propio embalse, que incluyeron perfiles de conductividad, determinación del total de sólidos disueltos, temperatura y salinidad.

Las mayores profundidades no sobrepasaron en ningún caso los ocho metros, condición que ha permitido una circulación vertical favorecida por el movimiento en superficie, producido por el viento, que contribuye a la homogeneización de la temperatura y la densidad. El perfil térmico durante el muestreo fue predominante en todo el embalse con una temperatura promedio de $26.4^{\circ} \mathrm{C}$, condiciones que lo clasifican como oligomítico, típico de lagos en regiones tropicales.

Los valores muestreados (tabla 1) revelaron una baja productividad primaria, según los bajos contenidos de nutrientes, poco significativos en nitritos y nitratos, aunque en dos estaciones se manifestaron actividades de amonio $\left(\mathrm{NH}_{4}\right)$; ello evidenció la baja producción de algas para este período de Iluvias, las cuales solo se observan en la zona de afluencia de la derivadora del río Salado y en la zona de transición, no así en la zona propiamente lacustre del embalse.

De acuerdo con los datos analizados, desde el punto de vista hidroquímico, se trata de aguas de poco tránsito, dulces y de muy baja mineralización, del tipo cloruradabicarbonatada-sódico-cálcica $\left(\mathrm{Cl}-\mathrm{HCO}_{3}-\mathrm{Na}-\mathrm{Ca}\right)$ 
que, específicamente en la cuenca vertiente y en el embalse, muestran valores mínimos de la relación cloruro-bicarabonato $\left(\mathrm{Cl} / \mathrm{HCO}_{3}\right)$ y que evidencian el aporte de una fuente de cloruros en las aguas de lluvia.

Tabla 1. Macroconstituyentes y nutrientes nitrogenados (en $\mathrm{mg} / \mathrm{L}$ ).

\begin{tabular}{lcccccccccccc}
\hline Estación & $\mathbf{p H}$ & $\mathbf{H C O}_{3}$ & $\mathbf{S O 4}$ & $\mathbf{C l}$ & $\mathbf{C a}$ & $\mathbf{M g}$ & $\mathbf{N a}$ & $\mathbf{K}$ & $\mathbf{N O}_{2}$ & $\mathbf{N O}_{3}$ & $\mathbf{N H}_{4}$ & $\mathrm{TDS}$ \\
\hline $\begin{array}{l}\text { Est.18 } \\
\text { Derivadora S }\end{array}$ & 6.53 & 18 & 13 & 14 & 7 & 2 & 8 & 3 & $<0.01$ & 1 & $<0.01$ & 65 \\
Est.5 Canal & & & & & & & & & & & & \\
Salado & 6.12 & 24 & 12 & 14 & 10 & 2 & 8 & 3 & $<0.01$ & 1 & $<0.01$ & 73 \\
Est.4 Cayo 1 & 7.41 & 24 & 7 & 11 & 10 & 1 & 5 & 2 & $<0.01$ & 1 & $\mathbf{0 . 0 4}$ & 60 \\
Est.10 & 6.28 & 24 & 7 & 11 & 7 & 2 & 5 & 2 & $<0.01$ & 1 & $<0.01$ & 58 \\
Est.2. & 7.27 & 24 & 7 & 7 & 6 & & 5 & 2 & $<0.01$ & 7 & $\mathbf{0 . 0 2}$ & 53 \\
Est. 30 Aliviadero & 6.88 & 24 & 7 & 11 & 8 & 2 & 5 & 2 & $<0.01$ & 1 & $<0.01$ & 59 \\
\hline LMA & & & $\mathbf{4 0 0}$ & $\mathbf{2 5 0}$ & $\mathbf{2 0 0}$ & $\mathbf{1 5 0}$ & $\mathbf{2 0 0}$ & & $\mathbf{0 . 0 1}$ & $\mathbf{4 5}$ & & $\mathbf{1 0 0 0}$ \\
\hline
\end{tabular}

Nota: LMA: Límite Máximo Admitido según (Oficina Nacional de Normalización [ONN], 2017).

Fuente: Elaboración propia.

Existe una amplia red lacustre natural y permanente de agua dulce y de lagunas costeras (humedales, manglares) a las que se integran depresiones que, en época de Iluvias, se tornan en pequeñas lagunas, así como canteras abandonadas (préstamos de material), devenidas en espejos de agua, ya consideradas bajo un criterio multidisciplinario como lagunas naturales y ecosistemas establecidos.

Los indicadores geofísicos de las 11 estaciones lacustres, muestreadas en mayo de 2018, se exponen en la tabla 2. La laguna Garnacha, según las características hidroquímicas identificadas, clasifica como aguas cloruradas sódicas $(\mathrm{Cl}-\mathrm{Na})$, patrón representativo en estos espejos de agua naturales cercanos a la costa.

Tabla 2. Valores geofisicos muestreados en lagunas costeras y de agua dulce.

\begin{tabular}{lcccc}
\hline \multicolumn{1}{c}{ Estación } & $\mathbf{T}^{\circ} \mathbf{C}$ & $\begin{array}{c}\text { SPC } \\
(\mathbf{m} \mathbf{m} / \mathbf{c m})\end{array}$ & $\begin{array}{c}\text { TDS } \\
(\mathbf{m g} / \mathbf{l})\end{array}$ & $\begin{array}{c}\text { Salinidad } \\
(\mathbf{m g} / \mathbf{l})\end{array}$ \\
\hline Est.2 Manglar 1 (cercano a Est.1) & & 11546 & 7494 & 6.55 \\
Est.6 Manglar 2 Camp. Pioneros & & 16140 & 10440 & 9.41 \\
Est.8 Laguna Préstamo & 31 & 97.3 & 64.36 & 0.04 \\
Est.9 Manglar 3 (Sur) & 33.8 & 62670 & 50755 & 41.92 \\
Est.10 Laguna Frente Camp. Pioneros & 26.9 & 162 & 105.95 & 0.08 \\
Est.11 Laguna Cantera Tarafa & 29 & 83.6 & 54.6 & 0.04 \\
Est.12 Laguna de la Marina & 31.5 & 717 & 468 & 0.35 \\
Est.16 Laguna Norte de Tarafa & 24.7 & 88 & 57.20 & 0.04 \\
Est.26 Laguna los Pozos & 26.4 & 8.5 & 54.6 & 0.04 \\
Est.27 Laguna Garnacha & 27.0 & 150.5 & 98.15 & 0.07 \\
Est.29 Laguna Manglar. Campismo viejo & 27.6 & 14338 & 9327 & 8.29 \\
\hline
\end{tabular}

Fuente: Elaboración propia. 
De acuerdo con los valores de conductividad (SPC), obtenidos en las aguas de las estaciones muestreadas, se pudieron diferenciar tres clases según la mineralización:

- Lagunas costeras que interactúan con el mar y presentan una mezcla de aguas con valores de conductividad (SPC) entre 11000 y $16000 \mathrm{~m} \mathrm{\mu cm}^{2}$.

- Lagunas con aguas poco profundas, sometidas a altas temperaturas que alcanzan valores de salmuera.

- Lagunas naturales interiores entre los 80 y $170 \mathrm{~m} \mathrm{ucm}^{2}$, muy cercanas a los valores del agua de lluvia.

\section{Aguas subterráneas}

En punta Colorada se han identificado dos horizontes acuíferos que dependen de las características litológicas de la zona. El primero de ellos a menos de $10 \mathrm{~m}$ de profundidad en depósitos poco consolidados, arenosos, de la Formación Guane. El segundo entre 25 y $35 \mathrm{~m}$ de profundidad asociado a las calizas organógenas y organógeno-detríticas de la Formación Paso Real, con aguas cársicas de muy buena calidad hidroquímica que se originaron a mucha distancia del área de estudio (Ortega, 2016).

De acuerdo con la información de las muestras tomadas en las fuentes más representativas de la zona de estudio, los valores de macroconstituyentes y nutrientes nitrogenados de las aguas subterráneas, solo en dos estaciones, sobrepasan el límite máximo admitido por la Norma Cubana de Agua Potable (ONN, 2017).
Las concentraciones de metales disueltos analizados responden a las características geológicas de la región (tabla 3), aunque llamaron la atención los valores de plomo $(\mathrm{Pb})$, cadmio $(\mathrm{Cd})$, cromo $(\mathrm{Cr})$ y vanadio $(V)$, detectados en la muestra de agua de mar correspondiente a la estación 4 punta Colorada, probablemente relacionados con la operación petrolera realizada en el área marítima, argumento que se ha considerado comprobar en estudios posteriores.

De igual forma resaltó la concentración de bario ( $\mathrm{Ba}$ ), detectada en la estación 19, considerada como valor anómalo por tratarse de un acueducto de abastecimiento al asentamiento Bolívar, lo que ha determinado repetir dicho análisis por no guardar relación con los valores comprobados en estaciones del mismo acuífero que sí constituyeron valores esperados.

Los patrones hidroquímicos, muy similares en las aguas de pozos asociados a las calizas de la Formación Paso Real, confirmaron la pertenencia de dichos pozos a un mismo acuífero, con aguas bicarbonatadas-cálcicas $\left(\mathrm{HCO}_{3}-\mathrm{Ca}\right)$. Las muestras analizadas expresaron una correlación bicarbonato/ cloruro menor que la unidad $\left(\mathrm{HCO}_{3} / \mathrm{Cl}<1\right)$, por lo tanto, consideradas como aguas poco afectadas por aguas de mar, lo cual ha fundamentado el diseño de abasto mediante una batería de pozos (Ortega, 2016) que presta particular atención a la calidad de las aguas y a las prácticas de explotación, históricamente ejecutadas, para el abasto a la población, los centros educacionales, las plantaciones frutales y agroforestales dispersas en toda la zona. 
Tabla 3. Metales y microlementos disueltos en las aguas de las estaciones muestreadas ( $\mathrm{mg} / \mathrm{l})$.

\begin{tabular}{lccccccccccc}
\hline Estación & $\mathbf{F e}$ & $\mathbf{~ N i}$ & $\mathbf{P b}$ & $\mathbf{Z n}$ & $\mathbf{C d}$ & $\mathbf{C r}$ & $\mathbf{C o}$ & $\mathbf{A g}$ & $\mathbf{B a}$ & $\mathbf{M o}$ & $\mathbf{V}$ \\
\hline Est.4 PC & $<0.07$ & $<0.02$ & $\mathbf{0 . 0 6}$ & $<0.02$ & $\mathbf{0 . 0 1}$ & $\mathbf{0 . 0 7}$ & $\mathbf{0 . 0 4}$ & $<0.02$ & $<0.02$ & $<0.02$ & $\mathbf{0 . 0 6}$ \\
Est.7 & $<0.07$ & $<0.02$ & $<0.03$ & $<0.02$ & $<0.003$ & $<0.003$ & $<0.007$ & $<0.02$ & $<0.02$ & $<0.02$ & $<0.003$ \\
Est.13 & $<0.07$ & $<0.02$ & $<0.03$ & $<0.02$ & $<0.003$ & $<0.003$ & $<0.007$ & $<0.02$ & $<0.02$ & $<0.02$ & $<0.003$ \\
Est.14 & $<0.07$ & $<0.02$ & $<0.03$ & $<0.02$ & $<0.003$ & $<0.003$ & $<0.007$ & $<0.02$ & 0.02 & $<0.02$ & $<0.003$ \\
Est.15 & $<0.07$ & $<0.02$ & $<0.03$ & $<0.02$ & $<0.003$ & $<0.003$ & $<0.007$ & $<0.02$ & $<0.02$ & $<0.02$ & $<0.003$ \\
Est.17 & $<0.07$ & $<0.02$ & $<0.03$ & $<0.02$ & $<0.003$ & $<0.003$ & $<0.007$ & $<0.02$ & $<0.02$ & $<0.02$ & $<0.003$ \\
Est.18 & $\mathbf{0 . 9}$ & $<0.02$ & $<0.03$ & $<0.02$ & $<0.003$ & $<0.003$ & $<0.007$ & $<0.02$ & $\mathbf{0 . 0 4}$ & $<0.02$ & $<0.003$ \\
Est.19 & $<0.07$ & $<0.02$ & $<0.03$ & $<0.02$ & $<0.003$ & $<0.003$ & $<0.007$ & $<0.02$ & $\mathbf{2 . 1}$ & $<0.02$ & $<0.003$ \\
Est.20 & $<0.07$ & $<0.02$ & $<0.03$ & $<0.02$ & $<0.003$ & $<0.003$ & $<0.007$ & $<0.02$ & $<0.02$ & $<0.02$ & $<0.003$ \\
Est. 21 & $<0.07$ & $<0.02$ & $<0.03$ & $<0.02$ & $<0.003$ & $<0.003$ & $<0.007$ & $<0.02$ & $<0.02$ & $<0.02$ & $<0.003$ \\
Est. 23 & $<0.07$ & $<0.02$ & $<0.03$ & $<0.02$ & $<0.003$ & $<0.003$ & $<0.007$ & $<0.02$ & $<0.02$ & $<0.02$ & $<0.003$ \\
Est.25 & $<0.07$ & $<0.02$ & $<0.03$ & $<0.02$ & $<0.003$ & $<0.003$ & $<0.007$ & $<0.02$ & 0.02 & $<0.02$ & $<0.003$ \\
Est.27 & $\mathbf{0 . 3}$ & $<0.02$ & $<0.03$ & $<0.02$ & $<0.003$ & $<0.003$ & $<0.007$ & $<0.02$ & $<0.02$ & $<0.02$ & $<0.003$ \\
Est. 30 & $<0.07$ & $<0.02$ & $<0.03$ & $<0.02$ & $<0.003$ & $<0.003$ & $<0.007$ & $<0.02$ & $<0.02$ & $<0.02$ & $<0.003$ \\
Est.2 LG & $<0.07$ & $<0.02$ & $<0.03$ & $<0.02$ & $<0.003$ & $<0.003$ & $<0.007$ & $<0.02$ & $<0.02$ & $<0.02$ & $<0.003$ \\
Est.4 LG & $<0.07$ & $<0.02$ & $<0.03$ & $<0.02$ & $<0.003$ & $<0.003$ & $<0.007$ & $<0.02$ & $<0.02$ & $<0.02$ & $<0.003$ \\
Est.5 LG & $\mathbf{0 . 2}$ & $<0.02$ & $<0.03$ & $<0.02$ & $<0.003$ & $<0.003$ & $<0.007$ & $<0.02$ & $\mathbf{0 . 0 4}$ & $<0.02$ & $<0.003$ \\
Est.10 & $<0.07$ & $<0.02$ & $<0.03$ & $<0.02$ & $<0.003$ & $<0.003$ & $<0.007$ & $<0,02$ & $<0.02$ & $<0.02$ & $<0.003$ \\
LG & & & & & & & & & &
\end{tabular}

Nota: LMA: Límite Máximo Admitido según (ONN, 2017).

Fuente: Elaboración propia.

Al respecto, el proyecto inversionista en el Máster Plan Punta Colorada ha considerado, como propuesta de abastecimiento, la construcción de cinco pozos concentrados de $40 \mathrm{~m}$ de profundidad en el sector norte del embalse Laguna Grande, con el fin de satisfacer la proyección de la demanda mediante el funcionamiento de cuatro pozos de manera permanente y uno de reserva, captación de hasta 18 horas, Io que puede provocar una sobrecarga al sistema acuífero.
Para el caso de los pozos ubicados a menos de $10 \mathrm{~m}$ de profundidad y asociados al escurrimiento de los depósitos poco consolidados arenosos y pisos de arcillas, de la Formación Guane, se identificaron patrones hidroquímicos de aguas bicarbonatadas-cloruradas-sódicas $\quad \mathrm{HCO}_{3}-$ $\mathrm{Cl}-\mathrm{Na}$ ) que confirman la presencia de otro acuífero (figura 5): 
Figura 5. Gráficos Scholler-Berkaloff-Molerio. Izquierda, pozos asociados al acuífero de la Formación Paso Real. Derecha, pozos asociados a la Formación Guane.
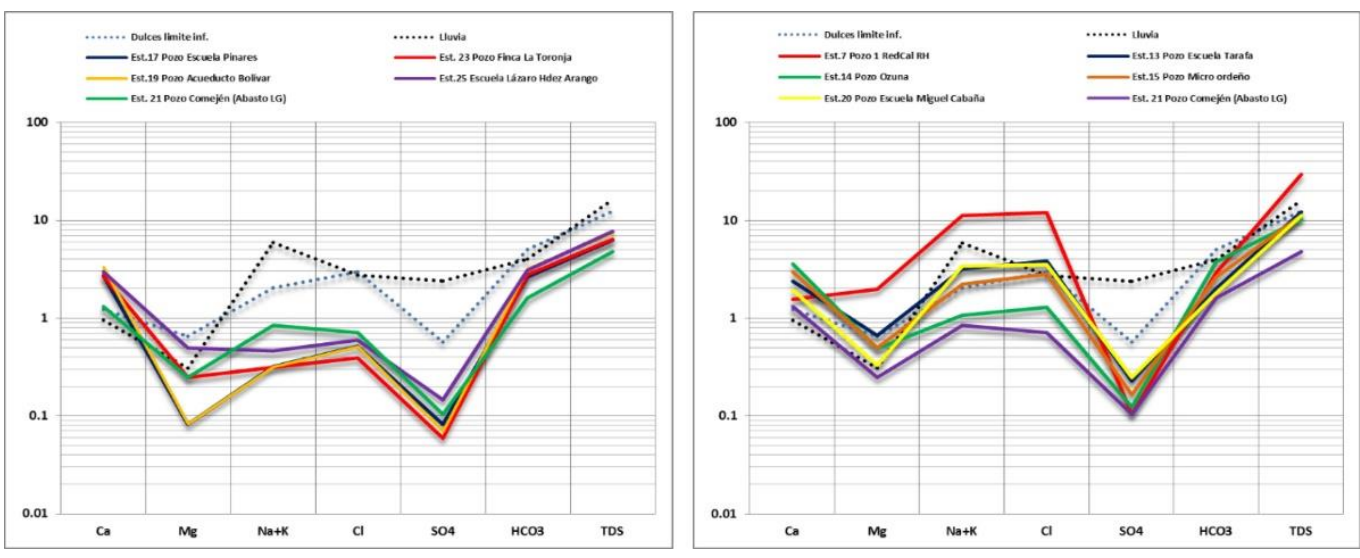

Fuente: Elaboración propia.

A partir del análisis del proyecto de inversiones descrito en el Máster Plan... (Planificación y Arquitectura, 2018), se han identificado objetos de obras con efectos ambientales negativos, tales como el canal interior que atraviesa la península, con acceso al mar en sus extremos norte, centro y sur, el cual fragmenta la línea de costa, el manglar asociado al litoral y los sectores de playas previstos entre las principales ofertas turísticas alternativas al golf (figura 6). Este canal ha sido diseñado como eje de movilidad interior y vía de acceso a diversas instalaciones e infraestructuras, por tanto, considerado como elemento esencial del proyecto; sin embargo, su construcción puede desencadenar una serie de efectos negativos importantes.

Figura 6. Esquema general del proyecto Punta Colorada (Fase I).

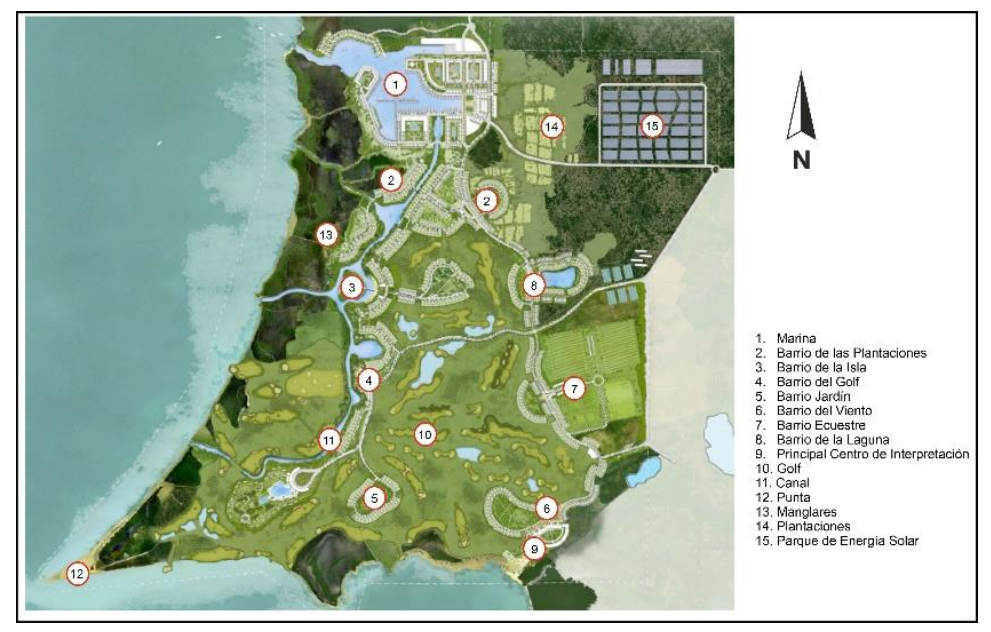

Fuente: Planificación y Arquitectura (2018). 
Igualmente, se han diseñado viales interiores, parcelas para la construcción de diversas obras de alojamiento y servicios, así como una marina para el atraque de yates en una laguna natural interior, los que requieren un considerable movimiento de tierras, cuantificado en un total de $1687.107 \mathrm{~m}^{3}$ por concepto de excavación.

Asociado a los anterior, se ha reconocido en este proyecto la modificación del suelo, la emisión de contaminantes (sólidos, líquidos y gases), la sobreexplotación de los recursos hídricos, la modificación del paisaje, entre otras, como las acciones con posibilidades de provocar cambios negativos, cuya mayor incidencia está en las aguas superficiales, subterráneas, marinas y su fauna. En tal sentido, específicamente relacionados con el recurso agua, se identificaron los siguientes impactos:

- Alteración del drenaje superficial y de la dinámica mar-tierra como consecuencia de la interrupción de las vías naturales de escurrimiento, debido al movimiento de tierra, la construcción de la Marina y del canal interior.

- Posibilidad de contaminación de las aguas superficiales y las subterráneas por derrames de hidrocarburos y lubricantes, por el mal manejo en el almacenamiento y durante el funcionamiento de los equipos y maquinarias, así como por la migración de contaminantes a partir de la acumulación de residuos sólidos y desechos peligrosos.

- Alteración de la hidrología del área, que constituye uno de los componentes básicos del humedal, que interactúa con el ambiente fisicoquímico del suelo, del agua y la biota.
- Modificación del equilibrio de la interface agua dulce-agua salada como consecuencia de la construcción de la Marina y el canal interior, lo cual provoca cambios sustanciales en el régimen y la calidad de las aguas subterráneas, previéndose una elevación sostenida de los valores de mineralización.

- Pérdida de la calidad del agua por mineralización y cambios en el régimen de las aguas subterráneas, por la sobreexplotación del acuífero como probable consecuencia de la concentración de la carga de extracción en cuatro pozos, para satisfacer la demanda del proyecto y su efecto combinado con la construcción del canal interior.

- Posibilidad de contaminación del agua marina por el arrastre de sedimentos desde el interior, debido al movimiento de tierra para la construcción de la Marina y el canal, así como por derrames de hidrocarburos y lubricantes, derivados de la operación de la draga para la habilitación del canal de entrada a esta.

En su diseño, el proyecto ha previsto actuaciones potencialmente positivas que dependen del uso y la aplicación correcta de los recursos y las tecnologías, así como del cumplimiento estricto de las correspondientes normas relacionadas con la gestión ambiental. Para tales fines se han considerado las siguientes acciones:

- Mantenimiento de las condiciones ambientales del área del proyecto por incremento del conocimiento científico y la apropiación de tecnologías limpias.

- Disminución de las afectaciones al medio ambiente a partir de la aplicación 
de buenas prácticas que incluyan la reutilización de recursos, la recuperación y el reciclaje de residuos, el uso de vehícul os eléctricos y de energías limpias.

- Sostenibilidad de las condiciones ambientales del área de influencia del proyecto mediante la sensibilización ambiental de los visitantes a través del funcionamiento del centro de interpretación.

\section{CONCLUSIONES Y RECOMENDACIONES}

La red de drenaje superficial descrita y el sistema de lagunas naturales constituyen elementos determinantes del soporte físico de los humedales presentes en ambas costas y su biota asociada. Desde el punto de vista hidrogeoquímico, las aguas clasifican como cloruradas-bicarbonatadas-sódico-cálcicas ( $\left.\mathrm{Cl}_{-} \mathrm{HCO}_{3}-\mathrm{Na}-\mathrm{Ca}\right)$ en el embalse Laguna Grande y como cloruradas sódicas $(\mathrm{Cl}-$ $\mathrm{Na}$ ), salobres y saladas, más próximo a la costa. Las aguas subterráneas propuestas para el abastecimiento clasifican como bicarbonatadas-cálcicas $\left(\mathrm{HCO}_{3}-\mathrm{Ca}\right)$ y aguas bicarbonatadas-cloruradas-sódicas $\left(\mathrm{HCO}_{3-}\right.$ $\mathrm{Cl}-\mathrm{Na})$.

La construcción de un canal interior en el área de estudio, como pieza básica en el engranaje conceptual del Máster
Plan..., significa una alteración sustancial al régimen y la calidad de las aguas tanto superficiales como subterráneas, en tanto modificará perceptible e irreversiblemente el equilibrio de la interface agua dulce-agua salada. La magnitud de este posible impacto negativo debe ser evaluada desde todos los ámbitos necesarios: biótico, abiótico y socioeconómico.

El diseño para el abasto de agua, propuesto por el Instituto Nacional de Recursos Hidráulicos, incluye más de 10 pozos ubicados en una amplia zona, que abarca dos cuencas subterráneas, para lograr una menor carga al sistema y establecer un régimen de explotación más racional. Sin embargo, la propuesta que se expone en el Máster Plan... concentra la carga de extracción en cuatro pozos al norte del embalse Laguna Grande, lo cual, unido a los efectos de la construcción del canal interior, deberá ser evaluado en términos de impacto.

Por la importancia del producto sol y playa para el proyecto, los valores de nutrientes nitrogenados y metales disueltos detectados en la muestra de agua de mar deberán ser objeto de estudios específicos.

\section{REFERENCIAS BIBLIOGRÁFICAS}

Balado, E.J. (2018). Descripción litológica de las arenas de punta Colorada (Informe Técnico). División de Ingeniería Ambiental y Recursos Hídricos. Inversiones GAMMA S.A.

Baños-Casteñeira, C.J., Vera-Rebollo, J.F. y Diéz-Santo, D. (2010). El abastecimiento de agua en los espacios y destinos turísticos de Alicante y Murcia. Investigaciones Geográficas, 51, 81-105. https://doi.org/10.14198/INGEO2010.51.04 
Díaz, A., Priestley, G., y Salgot, M. (2016, 16-18 noviembre). Agua para el desarrollo del turismo: una relación desde el nexus agua-cambio climático [Ponencia]. International Conference on Regional Science, Santiago de Compostela, España. https://bit. ly/3peMkkV

Dorticós, P.L., Arellano, M., y García, J.M.(Eds.). (2012). Los recursos hídricos en Cuba: una visión. En B. Jiménez y J. Galizia (Coord.), Diagnóstico del agua en las Américas (pp. 245-265). Foro Consultivo Científico y Tecnológico, AC. https://bit.ly/2HWBqju

Inversiones Gamma. (2018). Línea Base Ambiental. Informe Técnico de Estudio de Impacto Ambiental del Proyecto punta Colorada Golf y Marina, Fase I. División de Ingeniería Ambiental y Recursos Hídricos.

Ibarra, C.M. (1978). Mapa geográfico general. Atlas de Cuba XX Aniversario del Triunfo de la Revolución Cubana. Instituto Cubano de Geodesia y Cartografía.

Oficina Nacional de Normalización (2017). Agua Potable-Requisitos Sanitarios (Norma Cubana 827/2017). https://bit.ly/3aYbT5V

Ortega, M.A. (2016). Abasto de agua. Punta Colorada Golf Marinas. Etapa: Ideas conceptuales. Informe Técnico. Empresa de Investigaciones y Proyectos Hidráulicos. Instituto Nacional de Recursos Hidráulicos.

Peñalver, L.L., Cabrera, M., Trujillo, H., Morales, H., Fundora, M., Pérez, J., Molerio, L., Guerra, M., y Pedroso, I. (2001, 16-20 marzo). Evolución paleoclimática y paleogeográfica de Cuba durante el Cuaternario [Ponencia]. IV Congreso de Geología y Minería. La Habana, Cuba. https://bit.ly/2TKnqvJ

Planificación y Arquitectura (2018). Máster Plan Punta Colorada. Programa de Proyecto. Informe Técnico. PDP London.

Portela, A.H., Díaz, J.L., Hernández, J.R., Magaz, A.R. y Blanco, P. (1989). Geomorfología. Nuevo Atlas Nacional de Cuba. Sección IV-3. Instituto de Geografía, Academia de Ciencias de Cuba.

Salinas, Ed., Salinas, Er., y Mundet, L. (2019). El turismo en Cuba: Desarrollo, retos y perspectivas. Rosa dos Ventos-Turismo e Hospitalidade,11(1), 50-65. https://bit. ly/2KuAcgX 